\title{
Feasibility of Diagrammatic Monte-Carlo based on weak-coupling expansion in asymptotically free theories: case study of $O(N)$ sigma-model in the large- $N$ limit
}

\author{
P. V. Buividovich* \\ Regensburg University, D-93053 Regensburg, Germany \\ E-mail: pavel.buividovichephysik.uni-regensburg.de
}

\begin{abstract}
We discuss the feasibility of applying Diagrammatic Monte-Carlo algorithms to the weakcoupling expansions of asymptotically free quantum field theories, taking the large- $N$ limit of the $O(N)$ sigma-model as the simplest example where exact results are available. We use stereographic mapping from the sphere to the real plane to set up the perturbation theory, which results in a small bare mass term proportional to the coupling $\lambda$. Counting the powers of coupling associated with higher-order interaction vertices, we arrive at the double-series representation for the dynamically generated mass gap in powers of both $\lambda$ and $\log (\lambda)$, which converges quite quickly to the exact non-perturbative answer. We also demonstrate that it is feasible to obtain the coefficients of these double series by a Monte-Carlo sampling in the space of Feynman diagrams. In particular, the sign problem of such sampling becomes milder at small $\lambda$, that is, close to the continuum limit.
\end{abstract}

The 33rd International Symposium on Lattice Field Theory

14 -18 July 2015

Kobe International Conference Center, Kobe, Japan*

\footnotetext{
* Speaker.

$\dagger$ This work was supported by the S. Kowalevskaja award from the Alexander von Humboldt foundation. The author is grateful to G. Dunne, T. Sulejmanpasic and M. Unsal for interesting and stimulating discussions.
} 


\section{Introduction}

Diagrammatic Monte-Carlo (DiagMC) algorithms [四] which stochastically sample strong- or weak-coupling expansion diagrams provide a useful alternative to the standard Monte-Carlo algorithms which are based on stochastic sampling of field configurations. In recent years, DiagMC algorithms attracted a lot of interest from lattice QCD community as a prospective tool for reducing the sign problem in lattice QCD simulations at finite chemical potential [ [ $]$, []]. So far, all attempts to apply DiagMC to non-Abelian lattice gauge theories are based on the few lowest orders of the strong-coupling expansion of the QCD partition function. This approach turns out to be very efficient, since already the lowest order of strong-coupling expansion captures quark confinement, a fundamental feature of QCD. However, these algorithms become in general inapplicable as one approaches the continuum limit, since one has to take into account more and more terms in the strong coupling expansion.

Unfortunately, up to now no efficient ways of automated and systematically improvable stochastic sampling of strong-coupling expansions in lattice systems with $S U(N)$-valued degrees of freedom (including lattice QCD) are known. Moreover, one can expect that at large orders of strong-coupling expansion some terms in the series become negative, thus leading to a (real) sign problem in DiagMC. This sign problem should become quite severe close to the continuum, where large factors proportional to negative powers of coupling should cancel to yield a small result close to unity (e.g. for the mean plaquette).

In this situation it seems tempting to devise DiagMC algorithms which are based on the conventional weak-coupling perturbation theory, where diagrammatic rules are comparatively easy to obtain. Before turning to real simulations of lattice field theories with non-Abelian degrees of freedom, in these Proceedings we discuss the feasibility of such approach on the simplest example of the exactly solvable $O(N)$ sigma-model on the lattice in the large- $N$ limit. At the leading order in $1 / N$ expansion, only Feynman diagrams of "cactus" topology contribute to the weak-coupling expansion of this model, which allows one to obtain high-order expansion coefficients by a simple recursive procedure. Our aim here is to study whether these coefficients could be in principle obtained by Monte-Carlo sampling in the space of Feynman diagrams.

Having started with such a motivation, we immediately face the following conceptual problem: in asymptotically free QFTs the mass gap is typically non-perturbative and has the form $m^{2} \sim$ $e^{-\beta_{0} / \lambda}$, where $\beta_{0}$ is the zeroth order term in the expansion of the beta-function and $\lambda$ is the t'Hooft coupling constant. This statement is true also in the large- $N$ limit, where the number of Feynman diagrams which contribute at a given order of $1 / N$ expansion is known to grow exponentially with diagram order. Now if at the leading order of the $1 / N$ expansion the contribution of all diagrams is finite, the result of summation over them should be analytic in $\lambda$ at least in some vicinity of $\lambda=0$. Thus the only way in which the non-perturbative scale can emerge is through IR divergences in some of the diagrams. However, DiagMC simulations would be problematic if not impossible with such divergences.

In these Proceedings we show that certain parameterizations of field variables remove IR divergences in a self-consistent way even from "undressed" Feynman diagrams by introducing a small bare mass proportional to the coupling $\lambda$. The resulting series, however, no longer have a conventional form of power series in $\lambda$, but are rather double series both in $\lambda$ and in $\log \lambda$. Here we 
take a closer look at the structure of such series and demonstrate numerically that they converge to the exact nonperturbative answer. We also show that the coefficients of these series can be obtained by a Monte-Carlo sampling in the space of Feynman diagrams, with the sign problem becoming milder in the continuum limit.

\section{Large- $N O(N)$ sigma-model in stereographic coordinates}

Field variables in the $O(N)$ sigma model are $N$-component unit vectors $n_{a x}, a=0 \ldots N-1$ attached to the sites of the two-dimensional square lattice, which we label by $x$. The Euclidean partition function is given by

$$
\mathscr{Z}=\int \mathscr{D} n_{x} \exp \left(-\frac{N}{2 \lambda} \sum_{x, y, a} D_{x y} n_{a x} n_{a y}\right),
$$

where $D_{x, y}=4 \delta_{x, y}-\sum_{\mu=1,2} \delta_{x, y+\hat{\mu}}-\sum_{\mu=1,2} \delta_{x, y-\hat{\mu}}$ is the lattice Laplacian. Superficially, in the weakcoupling limit $\lambda \rightarrow 0$ all the vectors $n_{x}$ should align in one direction, thus spontaneously breaking the global $O(N)$ symmetry of the model and leaving $N-1$ massless Goldstone modes in the spectrum. However, by virtue of the Mermin-Wagner theorem such spontaneous symmetry breaking cannot occur in two dimensions, and the $O(N)$ symmetry remains unbroken for all values of $\lambda$.

The standard way to arrive at the weak-coupling perturbative expansion is, however, to expand around the minimum-energy configuration with all $n_{x}$ aligned in one direction, say, in the direction with $a=0$. In order to perform such expansion, we need to introduce some coordinates $\phi_{i x}, i=$ $1 \ldots N-1$ parameterizing the vectors $n_{x}$, such that $\phi_{i x}=0$ corresponds to the minimum-energy configuration with $n_{x}=$ const. In this work, we use the stereographic mapping

$$
n_{0 x}=\frac{1-\frac{\lambda}{4} \phi_{x}^{2}}{1+\frac{\lambda}{4} \phi_{x}^{2}}, \quad n_{i x}=\frac{\sqrt{\lambda} \phi_{i x}}{1+\frac{\lambda}{4} \phi_{x}^{2}}, \quad \phi_{x}^{2} \equiv \sum_{i} \phi_{i x} \phi_{i x}
$$

from the whole real space $\mathbb{R}^{N-1}$ to the whole sphere $S_{N}$. The integration measure on $S_{N}$ in terms of stereographic coordinates $\phi$ reads

$$
\mathscr{D} n_{x}=\mathscr{D} \phi_{x}\left(1+\frac{\lambda}{4} \phi_{x}^{2}\right)^{-N}
$$

Using ([2.2) and ([2.3), we can express the partition function as an integral over the fields $\phi_{x}$. In order to carry out the perturbative expansion, it is convenient to explicitly separate the "free" part of the action which is quadratic in $\phi_{x}$ from the interaction part, which contains higher powers of $\phi_{x}$ multiplied by some positive powers of the coupling constant $\lambda$ :

$$
\begin{gathered}
\mathscr{Z}=\int \mathscr{D} \phi_{x} \exp \left(-N \log \left(1+\frac{\lambda}{4} \phi_{x}^{2}\right)-\frac{N}{2 \lambda} \sum_{x, y} D_{x y} \frac{\left(1-\frac{\lambda}{4} \phi_{x}^{2}\right)\left(1-\frac{\lambda}{4} \phi_{y}^{2}\right)+\lambda\left(\phi_{x} \cdot \phi_{y}\right)}{\left(1+\frac{\lambda}{4} \phi_{x}^{2}\right)\left(1+\frac{\lambda}{4} \phi_{y}^{2}\right)}\right)= \\
=\int \mathscr{D} \phi_{x} \exp \left(-\frac{1}{2} \sum_{x, y}\left(D_{x y}+\frac{\lambda}{2} \delta_{x y}\right) \phi_{x} \cdot \phi_{y}+S_{I}[\phi]\right), \\
S_{I}[\phi]=\sum_{\substack{k, l=0 \\
k+l \neq 0}}^{+\infty} \frac{(-1)^{k+l} \lambda^{k+l}}{2 \cdot 4^{k+l}} \sum_{x, y} D_{x y}\left(\phi_{x}^{2}\right)^{k}\left(\phi_{y}^{2}\right)^{l}\left(\phi_{x} \cdot \phi_{y}\right)+\sum_{k=2}^{+\infty} \frac{(-1)^{k-1} \lambda^{k}}{4^{k} k} \sum_{x}\left(\phi_{x}^{2}\right)^{k},
\end{gathered}
$$


where $\phi_{x} \cdot \phi_{y} \equiv \sum_{i} \phi_{i x} \cdot \phi_{i y}$ and in the last expression for $S_{I}[\phi]$ we have dropped the summands of the form $\sum_{x, y} D_{x y}\left(\phi_{x}^{2}\right)^{k}\left(\phi_{y}^{2}\right)^{l}$ which are effectively zero in the large- $N$ limit by virtue of factorization and translational invariance. We thus see that due to the nontrivial integration measure in terms of the fields $\phi_{x}$ the bare "mass term" $\lambda / 2$ appears in the quadratic part of the action.

Having written the partition function and the action in the form ([2.4), now we can regard our $O(N)$ sigma-model simply as a large- $N$ quantum scalar field theory with infinitely many interaction vertices. The standard way to the exact solution of such theories is to consider the Schwinger-Dyson equations, which in the large- $N$ limit reduce to a single equation on the twopoint correlation function $\left\langle\phi_{x} \cdot \phi_{y}\right\rangle$. All correlators with larger number of field operators reduce to the products of $\left\langle\phi_{x} \cdot \phi_{y}\right\rangle$. Using the momentum-space representation of the two-point function $\left\langle\phi_{x} \cdot \phi_{y}\right\rangle=\int \frac{d^{2} p}{(2 \pi)^{2}} G(p) e^{i p(x-y)}$, where integration goes over the square Brillouin zone $p_{1}, p_{2} \in[-\pi, \pi]$, we can write the Schwinger-Dyson equations as

$$
G^{-1}(p)=D(p)+\sigma_{0}-D(p) \frac{\xi(2+\xi)}{(1+\xi)^{2}}-\frac{\lambda}{2} \frac{\xi}{1+\xi}-\frac{\lambda}{2} \frac{1}{(1+\xi)^{3}}[D G]_{x x},
$$

where $D(p)=4 \sin ^{2}\left(p_{1} / 2\right)+4 \sin ^{2}\left(p_{2} / 2\right)$ is the momentum-space representation of the lattice Laplacian in (2.]) and we have denoted $\sigma_{0} \equiv \lambda / 2$ and $\xi \equiv \frac{\lambda}{4}\left\langle\phi_{x}^{2}\right\rangle=\frac{\lambda}{4} \int \frac{d^{2} p}{(2 \pi)^{2}} G(p)$ and $[D G]_{x x}=$ $\int \frac{d^{2} p}{(2 \pi)^{2}} D(p) G(p)$. The notation $\sigma_{0} \equiv \lambda / 2$ is introduced to facilitate the formal counting of the positive powers of $\lambda$ associated with the vertices (rather than lines) of the Feynman diagrams, which we perform in the next Section.

From the form of the equation (2.5) one can immediately conclude that the momentum-space two-point function $G(p)$ should have the form of the free scalar field propagator with the wavefunction renormalization factor $z^{2}(\lambda)$ and the self-energy $\sigma(\lambda): G(p ; \lambda)=\frac{z^{2}(\lambda)}{D(p)+\sigma(\lambda)}$. From the Schwinger-Dyson equations ([2.5) one can readily deduce the following equations for $z$ and $\sigma$ :

$$
\sigma=\sigma_{0} z^{2}-\frac{\lambda}{2} z^{2}+\frac{\lambda}{2} z \sigma I_{0}(\sigma), \quad z=1+\frac{\lambda}{4} z^{2} I_{0}(\sigma),
$$

where we have defined $I_{0}(\sigma)=\int \frac{d^{2} p}{(2 \pi)^{2}} \frac{1}{D(p)+\sigma}=-\frac{1}{4 \pi} \log \left(\frac{\sigma}{32}\right)(1+O(\sigma))$. The last equality holds for sufficiently small values of $\sigma$, and we will use the latter expression with $O(\sigma)$ terms omitted. Substituting $\sigma_{0}=\lambda / 2$ into the equations (․, $)$, we obtain the following exact solutions for $z(\lambda)$ and $\sigma(\lambda)$ :

$$
z(\lambda)=2, \quad \lambda I_{0}(\sigma)=1 \Rightarrow \sigma(\lambda)=32 \exp \left(-\frac{4 \pi}{\lambda}\right)
$$

which explicitly demonstrates that the dynamically generated mass term $m(\lambda)=\sqrt{\sigma(\lambda)}$ is nonperturbative in the coupling constant $\lambda$.

\section{Formal perturbative expansion using stereographic coordinates}

The standard way to appear at the perturbative expansion is to expand the exponent of the interacting part of the action in power series and to explicitly integrate the resulting polynomials of field variables with the Gaussian weight containing the free part of the action. . 
In this work, we regard the term $\frac{\lambda}{2} \phi_{x}^{2}$ as a part of the free action, so that the free propagator becomes massive and thus IR divergences in perturbation theory are cured. In order to avoid any ambiguities in power counting, let us for the moment forget about the relation between $\sigma_{0}$ and $\lambda$ in the Schwinger-Dyson equations ([2.5)). We are now looking for the solution in form of the formal power series in $\lambda$ :

$$
z(\lambda)=\sum_{k=0}^{+\infty} z_{k}\left(\sigma_{0}\right) \lambda^{k}, \quad \sigma(\lambda)=\sum_{k=0}^{+\infty} \sigma_{k}\left(\sigma_{0}\right) \lambda^{k}
$$

with $z_{0}=1$. From the above equations one can recursively express $z_{k}\left(\sigma_{0}\right)$ and $\sigma_{k}\left(\sigma_{0}\right)$ in terms of all lower-order coefficients $z_{l}\left(\sigma_{0}\right), \sigma_{l}\left(\sigma_{0}\right)$ with $l<k$. Such recursion is mathematically equivalent to the summation of all the Feynman diagrams of a given order which contribute to the two-point function $G(p)$. In the process of recursion, we still treat $\sigma_{0}$ as a parameter independent of $\lambda$.
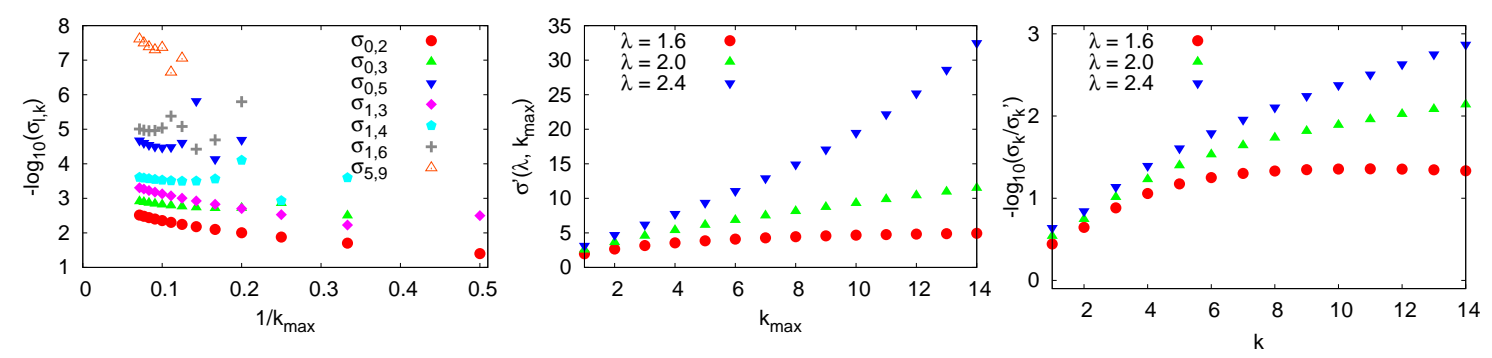

Figure 1: On the left: Dependence of the coefficients $\sigma_{k, l}$ in the double series (B.3) on the truncation order $k_{\max }$ in the formal power series (B.]). In the center: result of summation over Feynman diagrams without taking into account their signs as a function of the truncation order $k_{\max }$. On the right: effect of sign cancellations in the coefficients $\sigma_{k}$ of the formal power series (B.D.D).

The coefficients $z_{k}\left(\sigma_{0}\right)$ and $\sigma_{k}\left(\sigma_{0}\right)$ are now finite-degree polynomials in $\sigma_{0}, \sigma_{0}^{-1}$ and $\log \left(\frac{\sigma_{0}}{32}\right)$ which can be represented in the following general form:

$$
\sigma_{k}\left(\sigma_{0}\right)=\sum_{l=0}^{k}\left(-\log \left(\frac{\sigma_{0}}{32}\right)\right)^{l}\left(\sum_{i=i_{\min }(k-l-2)}^{\min (l, 1)} c_{k, l, i} \sigma_{0}^{i}\right), \quad i_{\min }(j)= \begin{cases}-j, & j \geq 0 \\ 0, & j=-1 \\ 1, & j=-2\end{cases}
$$

where each monomial term at fixed $k, l, i$ corresponds to a certain "cactus"-like diagram.

We obtain the coefficients $\sigma_{k}\left(\sigma_{0}\right)$ up to some finite order $k_{\max }$ using automated symbolic algebra and truncate the series (B. $)$ by summing over all orders $k$ up to $k_{\max }$. After that, we substitute $\sigma_{0} \rightarrow \lambda / 2$. As a result, we obtain the truncated double series in powers of both $\lambda$ and $\log (\lambda)$ of the form

$$
\sigma\left(\lambda, k_{\max }\right)=\sum_{l=0}^{k_{\max }}\left(-\log \left(\frac{\lambda}{64}\right)\right)^{l} \sum_{k=\min \left(l+2, k_{\max }\right)}^{k_{\max }+\min (l, 1)} \sigma_{l, k} \lambda^{k},
$$

and similarly for $z\left(\lambda, k_{\max }\right)$. It is important to stress that since upon the substitution $\sigma_{0} \rightarrow \lambda / 2$ the coefficients $\sigma_{k}\left(\sigma_{0}\right)$ contain also negative powers of $\lambda$, even coefficients $\sigma_{k}\left(\sigma_{0}\right)$ with large $k$ contribute to the coefficients $\sigma_{l, k}$ with small $k$ in the expansion (B.3), including the ones with $k=0$. In order to check whether the coefficients $\sigma_{k, l}$ have some well-defined limit as $k_{\max } \rightarrow \infty$, on the 
leftmost plot in Fig. W we show the dependence of some of these coefficients on $1 / k_{\max }$ at fixed $k$ and $l$. It seems that at least the few lowest-order coefficients do converge to certain limits at $k_{\max } \rightarrow \infty$.

Next, we check how well do the series (B. 1 ) truncated at the finite order $k_{\max }$ approximate the exact results ([2.]). To this end on Fig. \ we compare the results of the summation of truncated series for the renormalization factor $z\left(\lambda, k_{\max }\right)$ and the renormalized mass $m\left(\lambda, k_{\max }\right)=\sqrt{\sigma\left(\lambda, k_{\max }\right)}$ with exact results (2.7). We see that the dynamically generated mass $m\left(\lambda, k_{\max }\right)$ converges quite quickly to the exact result, and linear extrapolation to $1 / k_{\max }=0$ is enough to reproduce it within several percents. On the other hand, for the renormalization factor $z\left(\lambda, k_{\max }\right)$ the convergence to the exact answer $z(\lambda)=2$ is not so fast, and linear extrapolation from finite $k_{\max } \sim O(10)$ is not enough to reproduce it with sufficiently good precision.
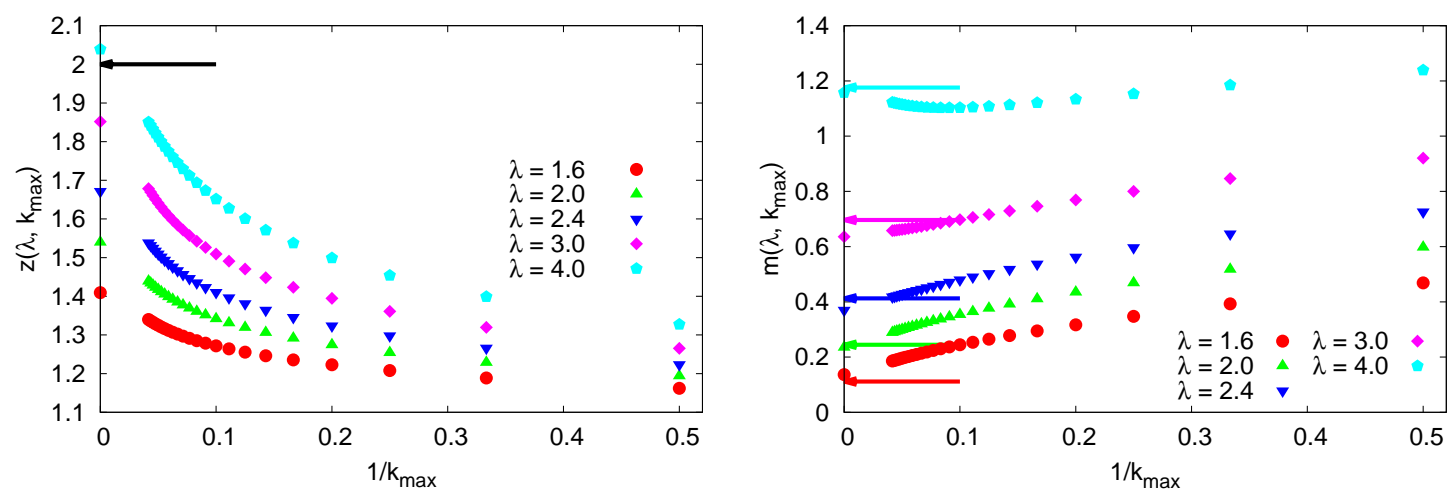

Figure 2: Dependence of the renormalization factor $z\left(\lambda, k_{\max }\right)$ (on the right) and the renormalized mass $m\left(\lambda, k_{\max }\right) \equiv \sqrt{\sigma\left(\lambda, k_{\max }\right)}$ (on the left) on the truncation order $k_{\max }$ in the formal power series (B. at $1 / k_{\text {max }}=0$ are the results of linear extrapolation from the numerical results with 7 largest values of $k_{\max }$. Arrows point to exact results (‥Z).

Let us now imagine, that we are not aware of the exact solution (2.7), and we try to find the coefficients $z_{k}\left(\sigma_{0}\right)$ and $\sigma_{k}\left(\sigma_{0}\right)$ in (B. لd ) by incorporating a generic DiagMC algorithm. Namely, we sample the bare Feynman diagrams with the probability proportional to their weight. For our model, the weights of individual "cactus"-like Feynman diagrams correspond to the coefficients of the monomial terms in (3.2). Since in our case these coefficient can be both positive and negative, for Monte-Carlo sampling we could take the absolute value of the diagram weight and treat the sign by reweighting. We denote the sum in (B.2) with all coefficients $c_{k, l, i}$ taken by absolute value as $\sigma_{k}^{\prime}\left(\sigma_{0}\right)$. Inserting the coefficients $\sigma_{k}^{\prime}\left(\sigma_{0}\right)$ into the series (B.D) instead of $\sigma_{k}\left(\sigma_{0}\right)$ and restricting the summation over $k$ to $k \leq k_{\max }$, we obtain the function $\sigma^{\prime}\left(\lambda, k_{\max }\right)$.

We first check whether the sum of the absolute values of diagram weights is finite in the limit $k_{\max } \rightarrow \infty$, so that individual weights can be interpreted as probabilities. To this end, on the central plot on Fig. $\square$ we plot $\sigma^{\prime}\left(\lambda, k_{\max }\right)$ as a function of $k_{\max }$. This plot indicates that $\sigma^{\prime}\left(\lambda, k_{\max }\right)$ has no finite limit at $k_{\max } \rightarrow \infty$, and the resulting double series are divergent unless one takes into account sign cancellations. In practice, however, this divergence can be quite easily circumvented by separately sampling the diagrams of different order and subsequent explicit summation.

The next question is then how strong are cancellations between same-order diagrams with 
positive and negative weights. To answer this question, on the rightmost plot on Fig. $\square$ we show the ratios of the coefficients $\sigma_{k}\left(\sigma_{0}\right)$ (where diagram weights retain their sign) to $\sigma_{k}^{\prime}\left(\sigma_{0}\right)$ (where the diagram weights are summed by absolute value). If this ratio is small, then sign cancellations are important. We see that the sign cancellations are quite important for high orders $k$, where the absolute values of $\sigma_{k}\left(\sigma_{0}\right)$ and $\sigma_{k}^{\prime}\left(\sigma_{0}\right)$ differ two or three orders of magnitude. However, at small values of $\lambda$ the sign cancellations become milder and only weakly depend on the diagram order $k$. Since small values of $\lambda$ correspond to the physically most interesting continuum limit, this is a very promising observation.

\section{Conclusions}

In these Proceedings, we have considered the weak-coupling perturbative expansion of the $O(N)$ sigma-model in the large- $N$ limit, taking the practical perspective of sampling Feynman diagrams by a generic DiagMC algorithm. In order to set up the perturbative expansion, we have used the stereographic mapping. As a result, bare propagators have acquired a small bare mass term proportional to the coupling $\lambda$. Counting only the positive powers of $\lambda$ associated with interaction vertices, we have arrived at the double series representation which involves powers of both $\lambda$ and $\log (\lambda)$. We have numerically checked the convergence of these series to the exact results, which turned out to be particularly fast for the dynamically generated mass gap and slower for the renormalization factor. Moreover, we have demonstrated that it is feasible to obtain the relevant series coefficients by a fictitious Monte-Carlo sampling in the space of bare Feynman diagrams. Interestingly, the sign problem which appears in such a sampling becomes milder as we approach the continuum limit at $\lambda \rightarrow 0$. These observations are very promising for further applications of DiagMC algorithms to asymptotically free field theories, most notably for the $U(N)$ principal chiral models and for non-Abelian gauge theories on the lattice.

\section{References}

[1] K. Van Houcke, E. Kozik, N. Prokof'ev, B. Svistunov, Diagrammatic Monte Carlo, in Computer Simulation Studies in Condensed Matter Physics XXI, Eds. D.P. Landau, S.P. Lewis, and H.B. Schuttler (Springer Verlag, Heidelberg, Berlin 2008) (2008), [0 802 . 2923].

[2] P. de Forcrand, M. Fromm, Nuclear physics from lattice QCD at strong coupling, Phys.Rev.Lett. 104 (2010) 112005, [0907.1915].

[3] M. Fromm, J. Langelage, S. Lottini, M. Neuman, O. Philipsen, Onset transition to cold nuclear matter from lattice QCD with heavy quarks, Phys.Rev.Lett. 110 (2013) 122001, [1207. 300 5]. 